\title{
Stereotactic radiofrequency thermocoagulation and resective surgery for patients with hypothalamic hamartoma
}

\author{
Shu Wang, MM, ${ }^{1}$ Meng Zhao, MD, ${ }^{1}$ Tianfu Li, MD, ${ }^{2-4}$ Chunsheng Zhang, MM, ${ }^{1}$ Jian Zhou, MD, ${ }^{1,3,4}$ \\ Mengyang Wang, MD, ${ }^{2}$ Xiongfei Wang, MD, ${ }^{1,3,4}$ Zhao Liu, MD, ${ }^{1}$ Kaiqiang Ma, MD, \\ Guoming Luan, MD, ${ }^{1,3,4}$ and Yuguang Guan, MD ${ }^{1,3,4}$
}

Departments of ${ }^{1}$ Neurosurgery and ${ }^{2}$ Neurology, SanBo Brain Hospital, Capital Medical University, Beijing; ${ }^{3}$ Beijing Key Laboratory of Epilepsy, Beijing; and ${ }^{4}$ Center of Epilepsy, Beijing Institute for Brain Disorders, Beijing, China

\begin{abstract}
OBJECTIVE In this study, the authors compared the efficacy and safety of stereotactic radiofrequency thermocoagulation (SRT) and resective surgery (RS) for patients with hypothalamic hamartoma $(\mathrm{HH})$.

METHODS The authors included all patients with HHs who were treated by SRT or hamartoma resection. Seizure outcomes were assessed by blinded observers according to the International League Against Epilepsy (ILAE) classification. Favorable seizure outcomes were defined as ILAE classes 1 and 2, and unfavorable seizure outcomes were defined as ILAE classes 3-6.

RESULTS Twenty-nine patients who underwent SRT or RS met the inclusion criteria; 3 were excluded because they had completed less than 12 months of follow-up. Most of the patients (20 of 26; 76.9\%) had small HHs (i.e., maximum $\mathrm{HH}$ diameter less than $20 \mathrm{~mm}$ ). The patients' follow-up time ranged from 12 to 66 months (median 60 months). At the last follow-up, favorable outcomes were observed in 9 patients (69.2\%) who had undergone SRT and 10 patients (76.9\%) who had undergone $\mathrm{HH}$ resection. No significant difference was found in seizure outcomes between SRT and RS recipients. Patients with giant HHs were more likely than patients with smaller tumors to undergo multiple resections ( $p$ $=0.043$, univariate logistic regression; significant). However, no significant difference was found between SRT and RS recipients in terms of the number of procedures per patient. SRT recipients had fewer and less-severe adverse events than $\mathrm{RS}$ recipients.
\end{abstract}

CONCLUSIONS For patients with small HHs, SRT provides similar seizure outcomes to RS with a less invasive procedure. Patients who underwent SRT experienced fewer and lighter adverse effects than patients who had RS. Patients with giant $\mathrm{HH}$ s were more likely to undergo multiple $\mathrm{HH}$ resections.

https://thejns.org/doi/abs/10.3171/2020.2.JNS193423

KEYWORDS hypothalamic hamartoma; stereotactic radiofrequency thermocoagulation; surgery; gelastic seizure; epilepsy; outcomes

$\mathrm{H}$ YPOTHALAMIC hamartoma $(\mathrm{HH})$ is a rare congenital, nonprogressive abnormality located in the ventral hypothalamus. HHs are intrinsically epileptogenic ${ }^{1-4}$ and are associated with several common comorbidities, such as cognitive delay, precocious puberty, and behavioral disorders. ${ }^{5-9}$ Gelastic seizures (GSs) are the typical seizure type for patients with $\mathrm{HHs},{ }^{3}$ but other seizure types, including complex partial seizures (CPSs), simple partial seizures (SPSs), and generalized tonic-clon- ic seizures (GTCSs), can also be observed in approximately $75 \%$ of patients. ${ }^{8,10,11} \mathrm{HH}$-related seizures are often resistant to antiepileptic drugs (AEDs). ${ }^{12}$ Surgical treatment of HHs is widely considered an essential step for seizure control. Several surgical approaches and techniques have been used to resect or disconnect HHs and have achieved effective seizure outcomes. ${ }^{13-18}$ However, some patients refuse to undergo resective surgery (RS); additionally, some HHs are positioned too deep to reach, and $37.9 \%-65.4 \%$

ABBREVIATIONS AED = antiepileptic drug; CPS = complex partial seizure; EEG = electroencephalography; GKRS = Gamma Knife radiosurgery; GS = gelastic seizure; GTCS = generalized tonic-clonic seizure; HH = hypothalamic hamartoma; ILAE = International League Against Epilepsy; LITT = laser interstitial thermal therapy; RS = resective surgery; SPS = simple partial seizure; SRT = stereotactic radiofrequency thermocoagulation.

SUBMITTED December 18, 2019. ACCEPTED February 13, 2020.

INCLUDE WHEN CITING Published online April 17, 2020; DOI: 10.3171/2020.2.JNS193423. 
of HHs cannot be completely or almost completely resected..$^{15,17,19}$ As a minimally invasive and highly effective surgical procedure, stereotactic radiofrequency thermocoagulation (SRT) is an increasingly common treatment for $\mathrm{HH} .{ }^{20-23}$ Although several studies have separately reported the use of SRT and RS for HHs, few studies have directly compared these 2 therapeutic methods. The present study compared the efficacy and safety of SRT and RS to determine which is the better choice for HH treatment. In addition, the present study supplements the existing literature on the long-term effectiveness of SRT and RS.

\section{Methods \\ Patient Population and Inclusion Criteria}

This single-blind study included all patients with $\mathrm{HH}$ who were treated by SRT or RS between 2009 and 2019 at SanBo Brain Hospital, Capital Medical University, Beijing, China. Written informed consent was provided by the patients or their parents. All patients agreed to the procedure and to the use of their anonymized data for research purposes. The local ethics committee approved this study. Preoperative evaluation included CT, MRI, concurrent video and electroencephalography (video-EEG), magnetoencephalography, and neuropsychological assessment. We established a unified anonymous medical record system to manage the characteristics, clinical histories, examination results, and follow-up outcomes of all patients. The inclusion criteria required all patients to have been clinically diagnosed with $\mathrm{HH}$ by a neurosurgeon based on MRI findings, syndromes, and pathology (if available) and to have undergone SRT or RS at SanBo Brain Hospital, Capital Medical University, between 2009 and 2019. All included patients had refractory epilepsy, defined as failure of at least 3 AEDs. Patients who completed less than 12 months of follow-up or refused to participate were excluded from the present study.

\section{Surgical Strategy}

Each patient's treatment strategy was developed according to surgeon recommendations based on the location, shape, and size of the $\mathrm{HH}$ and the condition of the patient. Placement of the SRT electrode was performed under general anesthesia guided by a ROSA robot (MedTech) or a Sinovation robot (Sinovation). ROSA planning software (MedTech) or Sinovation planning software (Sinovation) was used for surgical planning based on an analysis of the MR images (1-mm-slice sagittal T1-weighted images and 2-mm-slice axial and coronal T2-weighted images; $1.5 \mathrm{~T}$ scanner, Siemens) and CT scans (1-mm consecutive, Philips). After the intracranial electrodes were implanted, additional MRI and CT scans were obtained to confirm their locations. Intracranial EEG recordings were monitored to locate the epileptogenic zone. SRT was performed on the contacts of the epileptogenic zone using a radiofrequency lesion generator (model R-2000B, BNS). A test heating (2 W, 30 seconds) was performed first, and the patient was monitored by EEG for 1 day. If the patient did not experience any unacceptable complications, we then created lesions ( $3 \mathrm{~W}, 60$ seconds). The coagulations were mainly focused at the border between the hamartoma and the hy- pothalamus and at the center of the hamartoma. After the first SRT session, the patient was monitored by EEG again for at least 1 night. Repeat SRT procedures were conducted if the patient's seizures persisted. The decision to end the course of SRT and resect the electrode was made based on the patient's condition, wishes, and seizures.

RS was also performed under general anesthesia. Standard transventricular resection of the $\mathrm{HH}$ was conducted for all patients. First, a frontal craniotomy was made along the optimal trajectory, which was determined by a navigation system. Then, an opening was made in the anterior corpus callosum under microscopic guidance, and the roof of the third ventricle was entered through the midline of the corpus callosum. The $\mathrm{HH}$ could be found in the lateral wall of the third ventricle. MRI and CT scans were obtained postoperatively to confirm the resection boundary. The patient was asked to return for assessment. Further resections were performed based on the patient's seizures, condition, and wishes. The pre- and postoperative MR images obtained in 2 patients with $\mathrm{HH}$ who underwent SRT (patient 10) and RS (patient 15) are shown in Fig. 1. Implantation planning is also shown in 1 patient (patient 10).

\section{Assessment}

Seizure outcomes were assessed by blinded observers according to the International League Against Epilepsy (ILAE) classification. ${ }^{24}$ ILAE class 1 means the patient is entirely free of seizures and auras, ILAE class 2 means the patient has only auras and no other seizures, and ILAE classes 3-6 mean the patient still has seizures with or without auras. Favorable seizure outcomes were defined as ILAE classes 1 and 2, and unfavorable seizure outcomes were defined as classes 3-6. Experienced neurosurgeons performed the assessments at baseline, 3 months, and every year after the procedure. The neurosurgeons were merely told a random number identifying the patient and entered the follow-up records in the anonymous medical record system. Seizure frequency at baseline was determined by averaging the number of seizures per month in the last 3 months before the implantation surgery. Other outcomes, including adverse events and AED usage, were also collected.

\section{Statistical Analysis}

Continuous variables are presented as the mean \pm SD. Categorical variables are reported as a number and percentage. After the Shapiro-Wilk test and Levene's test were used to explore the distributions and variance, the 2-sample t-test or Mann-Whitney U-test was used to compare the continuous variables (e.g., patient age). Univariate analyses and comparisons of categorical variables (e.g., favorable or unfavorable outcomes) were performed using Fisher's exact test and logistic regression. Statistical significance was defined as $\mathrm{p}<0.05$. Statistical analyses were performed using IBM SPSS (version 26, IBM Corp.); statistical figures were drawn using GraphPad Prism (version 8, GraphPad).

\section{Results}

\section{Baseline Characteristics of Patients}

Twenty-nine patients who underwent SRT or RS met 

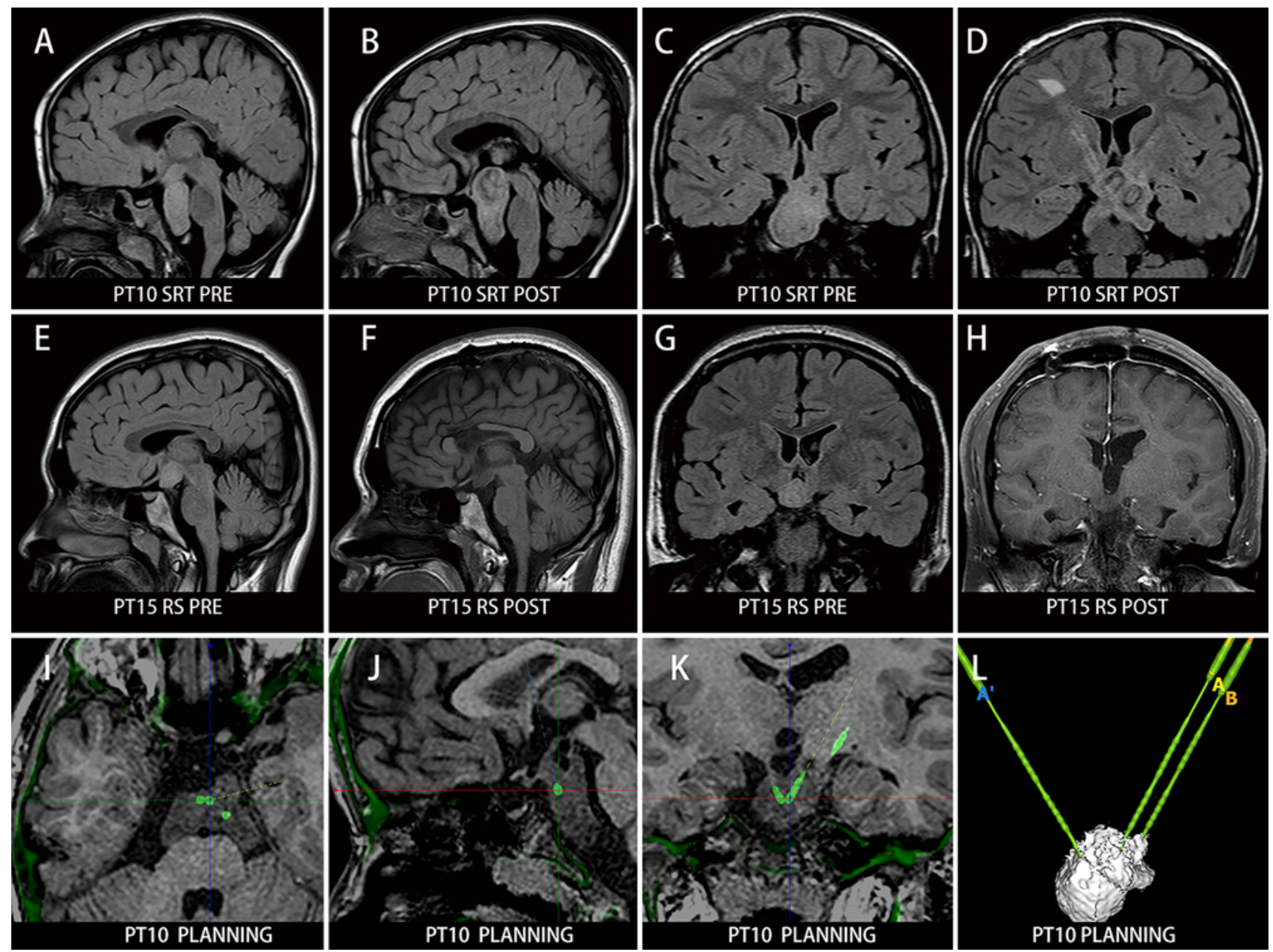

FIG. 1. Preoperative and postoperative MR images and implantation planning in 2 patients with $\mathrm{HH}$ who underwent SRT (patient 10) and RS (patient 15). A-D: Preoperative (A) and postoperative (B) sagittal FLAIR images, and preoperative (C) and postoperative (D) coronal FLAIR images. E-H: Patient 15. Preoperative (E) and postoperative (F) sagittal FLAIR images, preoperative coronal FLAIR image (G), and postoperative contrast-enhanced coronal T1-weighted image (H). I-L: Patient 10. Axial (I) and sagittal $(\mathrm{J})$ implantation planning images, coronal implantation planning image $(\mathrm{K})$, and 3D reconstructed image of implantation planning $(\mathrm{L})$. Figure is available in color online only.

the inclusion criteria; 3 of them were excluded because they had completed less than 12 months of follow-up. The remaining 26 patients (18 male and 8 female patients) had been diagnosed with $\mathrm{HH}$ and all agreed to participate in this study. These patients were divided into the SRT group (13 patients, 50\%) and the RS group (13 patients, 50\%), which determined the type of treatment they would undergo at our epilepsy center. The clinical characteristics of these patients and the treatments they received are described in Table 1.

The patients' follow-up time ranged from 12 to 66 months (median 60 months). Most of the patients (20 of 26; $76.9 \%$ ) had small $\mathrm{HHs}$ (i.e., maximum $\mathrm{HH}$ diameter less than $20 \mathrm{~mm}$ ). Three patients (11.5\%) had experienced only GSs before; other seizure types included CPS, SPS, and GTCS. There was no significant difference in sex, age, $\mathrm{HH}$ size, symptom history, follow-up time, or number of procedures between patients who underwent SRT and those who underwent RS ( $p>0.05$; Fisher's exact test, 2-sample t-test, or Mann-Whitney U-test, as appropriate). Of the 13 patients who underwent SRT, 1 patient (patient 4) had previously undergone Gamma Knife radiosurgery (GKRS), and 1 patient (patient 8) had previously undergone $\mathrm{HH}$ resection at another medical center. Seven patients (53.8\%) received SRT more than once. As for patients who underwent RS, 1 patient (patient 14) had a history of GKRS. Moreover, 1 patient (patient 16) switched to SRT after 2 $\mathrm{HH}$ resections.

\section{SRT and RS Procedures}

Among patients who underwent SRT, 53.8\% (7 of 13 patients) received multiple SRT procedures. Most of them (6 of 7 patients, 85.7\%) received SRT twice, and 1 patient had the procedure 4 times, eventually achieving a seizurefree outcome. As for the RS group, full resection was not achieved in 5 patients on the first attempt; 4 of them underwent a second resection. One patient received SRT after 2 RSs and had an ILAE class of 4 at the last follow-up. The procedures and outcomes of SRT and RS are shown in Fig. 2. To determine the factors that influence the proce- 
TABLE 1. Characteristics and treatments of patients with $\mathrm{HH}$

\begin{tabular}{|c|c|c|c|c|c|c|c|c|c|}
\hline Patient No. & Sex & Age (yrs) & Size (mm) & History (yrs) & Seizure Type & Comorbidities & FU (mos) & Previous Tx & $\begin{array}{c}\text { No. of } \\
\text { Procedures }\end{array}$ \\
\hline \multicolumn{10}{|l|}{ SRT group } \\
\hline 1 & $\mathrm{~F}$ & 14 & 8.8 & 14 & GS, SPS & & 54 & None & 1 \\
\hline 2 & M & 7 & 7 & 5 & GS, CPS & & 60 & None & 1 \\
\hline 3 & M & 10 & 8.9 & 9 & GS, CPS & & 60 & None & 1 \\
\hline 4 & M & 23 & 10 & 21 & GS & & 54 & GKRS & 2 \\
\hline 5 & $M$ & 9 & 13 & 4 & GS, SPS & $\mathrm{CD}, \mathrm{PP}$ & 60 & None & 2 \\
\hline 6 & $M$ & 4 & 9 & 2.5 & GS, SPS & & 60 & None & 2 \\
\hline 7 & $M$ & 25 & 13 & 5 & GS, CPS & & 60 & None & 1 \\
\hline 8 & $M$ & 19 & 6.2 & 18 & GS, SPS & $B D$ & 60 & RS & 1 \\
\hline 9 & $\mathrm{~F}$ & 2 & 17 & 0.5 & GS & PP & 60 & None & 4 \\
\hline 10 & M & 4 & 32 & 2 & GS, SPS & & 54 & None & 1 \\
\hline 11 & $\mathrm{~F}$ & 4 & 33 & 0.58 & GS, CPS & & 12 & None & 2 \\
\hline 12 & $\mathrm{~F}$ & 10 & 5.5 & 0.5 & GS, CPS, GTCS & & 54 & None & 2 \\
\hline 13 & $\mathrm{~F}$ & 24 & 11.5 & 4 & GS, GTCS & & 12 & None & 2 \\
\hline Mean & & 11.92 & 13.45 & 6.62 & & & 50.77 & & 1.69 \\
\hline \multicolumn{10}{|l|}{ RS group } \\
\hline 14 & $M$ & 36 & 30 & 34 & GS, CPS, GTCS & $\mathrm{BD}$ & 66 & GKRS & 1 \\
\hline 15 & $M$ & 15 & 16 & 14 & GS, SPS & & 60 & None & 1 \\
\hline 16 & $\mathrm{~F}$ & 3 & 31 & 2 & GS & PP & 54 & None $^{*}$ & 2 \\
\hline 17 & $M$ & 9 & 26 & 0.42 & GS, GTCS & & 30 & None & 2 \\
\hline 18 & $M$ & 14 & 10 & 13 & GS, CPS, GTCS & PP & 66 & None & 1 \\
\hline 19 & M & 5 & 11 & 0.75 & GS, CPS & $\mathrm{CD}, \mathrm{BD}$ & 66 & None & 2 \\
\hline 20 & $\mathrm{~F}$ & 20 & 7 & 18 & GS, SPS & & 60 & None & 1 \\
\hline 21 & $M$ & 16 & 7 & 8 & GS, SPS & & 60 & None & 1 \\
\hline 22 & $\mathrm{~F}$ & 27 & 20 & 13 & GS, GTCS & PP, BD & 60 & None & 2 \\
\hline 23 & $M$ & 35 & 7.4 & 34 & GS, SPS & & 24 & None & 1 \\
\hline 24 & M & 20 & 6 & 20 & GS, GTCS & $C D$ & 60 & None & 1 \\
\hline 25 & $M$ & 31 & 11.5 & 3 & GS, CPS & & 60 & None & 1 \\
\hline 26 & M & 14 & 11 & 14 & GS, SPS, GTCS & & 60 & None & 1 \\
\hline Mean & & 18.85 & 14.92 & 13.40 & & & 55.85 & & 1.31 \\
\hline$p$ value & 0.51 & 0.08 & 0.76 & 0.19 & & & 0.14 & & 0.29 \\
\hline
\end{tabular}

$\mathrm{BD}=$ behavioral disorders; $\mathrm{CD}=$ cognitive delay; $\mathrm{FU}=$ follow-up; $\mathrm{PP}=$ precocious puberty; $\mathrm{Tx}=$ treatment.

* This patient underwent SRT after 2 RSs.

dure times, we conducted comparisons and predictors of SRT and RS times by univariate analysis (Supplementary Table S1). Patients with giant HHs (i.e., maximum HH diameter more than $20 \mathrm{~mm}$ ) were significantly more likely than those with smaller HHs to undergo multiple resections (OR 3.00, 95\% CI 1.97-8.30; $p=0.043$, univariate logistic regression); no other predictors of the number of SRT or RS procedures were found. Moreover, an analysis of different subgroups did not reveal any significant difference between the re-treatment rates of SRT and RS (53.8\% vs $30.8 \%$ with multiple surgeries).

\section{Seizure Outcomes}

At the last follow-up, favorable outcomes were observed in 9 patients $(69.2 \%)$ who had undergone SRT and 10 patients (76.9\%) who had undergone RS. No significant difference was found between SRT and RS outcomes. In univariate analysis, no predictors were associated with seizure outcomes (Supplementary Table S2). The RS group initially achieved a higher seizure-free rate than the SRT group, but the SRT group surpassed the RS group at 6 and 12 months. Over long-term follow-up, the seizure-free rate of the RS group gradually increased and eventually exceeded that of the SRT group (Fig. 3). During the followup period, the difference in seizure-free rates between the SRT and RS groups was not significant ( $p>0.05$, Fisher's exact test).

The adverse effects in these 2 groups were also recorded (Table 2) and compared (Supplementary Table S3). Among SRT recipients, hypothyroidism was observed in 2 patients (15.4\%; 1 single SRT and 1 multiple SRTs); diminished short-term memory was reported by 1 patient (7.7\%, multiple SRTs); and 1 patient (7.7\%, multiple SRTs) had weight gain. As for the RS group, replacement therapy 


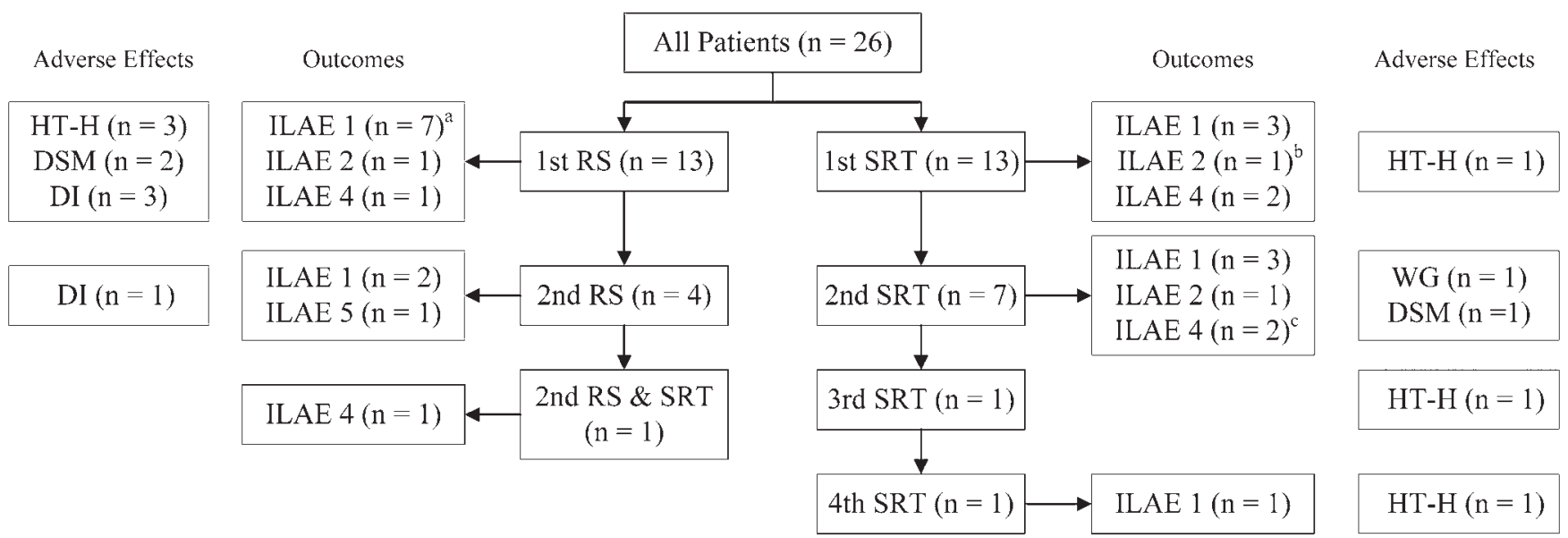

FIG. 2. Flow diagram showing the repeat procedures and outcomes of SRT and RS for patients with $H H$, and the adverse effects of each procedure. ${ }^{\mathrm{a}}$ One patient had a history of GKRS; ${ }^{b}$ one patient had a history of $\mathrm{HH}$ resection; ${ }^{\mathrm{c}}$ one patient had a history of GKRS. DI = diabetes insipidus; $\mathrm{DSM}=$ diminished short-term memory; $\mathrm{HT}-\mathrm{H}=$ hypothyroidism; $\mathrm{WG}$ = weight gain.

for hypothyroidism was required by 3 patients $(23.1 \%$, all single RSs); diminished short-term memory was reported by 2 patients (15.4\%, both single RSs), and diabetes insipidus was found in 4 patients $(30.8 \%, 3$ with single RSs and 1 with multiple RSs). The dose of AEDs was reduced in 2 patients who received SRT $(15.4 \%, 1$ with single SRT and 1 with multiple SRTs) and 3 patients with RS (23.1\%, all single RSs).

\section{Discussion \\ Efficacy of SRT and RS}

Several previous studies separately reported on SRT and RS as treatments for $\mathrm{HH}$, including the efficacy of these treatments and the associated adverse events. However, the number of comparisons between these two therapeutic methods has been limited. The present study compared SRT with RS in terms of efficacy and adverse events to determine which is the better choice for $\mathrm{HH}$. As most of the included patients had small $\mathrm{HHs}$, the results might be more applicable to patients with small HHs than to those with larger tumors.

SRT was previously reported by several studies as a minimally invasive and effective procedure for $\mathrm{HH} .{ }^{20-23}$, ${ }^{25,26}$ Kameyama et al..$^{20,21}$ treated HH by conducting MRIguided SRT procedures using a stereotactic system. They reviewed 100 patients with a median follow-up time of 36 months and reported that complete freedom from seizures was achieved in $71.0 \%$ of patients. In the present study, we performed SRT with the guidance of a ROSA or Sinovation robot in accordance with the technical note of Tandon et al. ${ }^{27}$ and made lesions based on findings from intracranial EEG recordings. Intracranial EEG could provide accurate localization of the epileptogenic focus and guidance for reoperation. In our study, 7 patients (53.8\%) achieved seizure-free outcomes and 9 patients (69.2\%) achieved favorable outcomes after a median follow-up period of 60 months in the SRT group.

Among patients who underwent one-time SRT, 66.67\% achieved favorable outcomes. Repeat procedures were needed in $53.8 \%$ of patients, and all of them achieved a lower seizure frequency after the additional procedure than after the first procedure. Kameyama et al. ${ }^{21}$ also reported that approximately $70 \%$ of patients achieved good therapeutic outcomes after a single SRT session, and repeat sessions could be effective. A smaller HH size and a smaller number of AEDs were believed to be associated with better neuropsychological performance in a study by Sonoda et al..$^{23}$ In the present study, however, no predictors were found to be associated with seizure outcomes in univariate analysis. The study of Kameyama et al. ${ }^{21}$ proposed that repeat SRT was ineffective for residual non-GSs. We also found that all patients with unfavorable outcomes had non-GSs as of the last follow-up. Residual non-GSs included SPSs $(n=2)$ and CPSs $(n=2)$. For patients with giant HHs, a study by Shirozu et al. ${ }^{22}$ showed that SRT could also be an acceptable strategy, which was confirmed in our 2 patients with giant HHs who underwent SRT. We attempted to compare different sizes of $\mathrm{HH}$ and found no

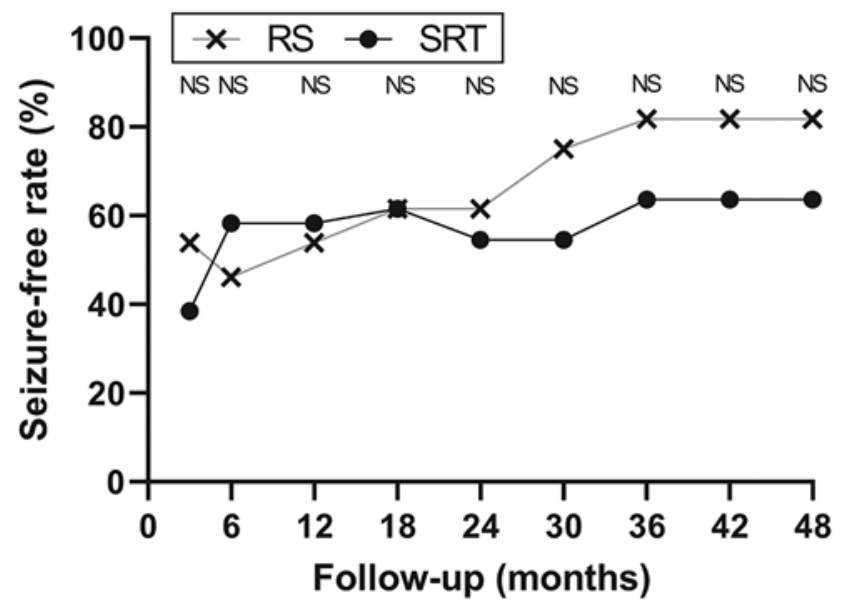

FIG. 3. Seizure-free rate of SRT and RS for patients with HH. NS = not significant. 
TABLE 2. Adverse effects resulting from SRT and RS for patients with HH

\begin{tabular}{ccccc}
\hline Patient No. & Procedure & No. of Procedures & Complication & Outcome \\
\hline 5 & SRT & 2 & Weight gain & Resolved \\
\hline 9 & SRT & 3 & Hypothyroidism & Replacement therapy \\
\hline 9 & SRT & 4 & Hypothyroidism & Replacement therapy \\
\hline 13 & SRT & 1 & Hypothyroidism & Resolved \\
\hline 13 & SRT & 2 & Diminished short-term memory & Resolved \\
\hline 15 & RS & 1 & Diabetes insipidus & Resolved \\
\hline 17 & RS & 1 & Diminished short-term memory & Resolved \\
\hline 17 & RS & 2 & Diabetes insipidus & Resolved \\
\hline 18 & RS & 1 & Hypothyroidism & Replacement therapy \\
\hline 19 & RS & 1 & Hypothyroidism & Replacement therapy \\
\hline 20 & RS & 1 & Hypothyroidism & Replacement therapy \\
\hline 24 & RS & 1 & Diabetes insipidus & Desmopressin Tx \\
\hline 25 & RS & 1 & Diabetes insipidus & Resolved \\
\hline 26 & RS & 1 & Diminished short-term memory & Resolved \\
\hline
\end{tabular}

difference in seizure outcomes between small and giant HHs.

Standard transventricular resection of $\mathrm{HHs}$ was conducted in the RS group. Several surgical approaches and techniques have been used for $\mathrm{HH}$ resection. Most of these methods have been reported to be safe and effective but are prone to a high probability of adverse events..$^{14-17,19}$ In the present study, 10 patients $(76.9 \%)$ underwent $\mathrm{HH}$ resections and achieved favorable outcomes; 9 patients (69.2\%) were free of seizures at the last follow-up. Harvey et al. ${ }^{15}$ conducted $\mathrm{HH}$ resections using a transcallosal approach and reported that 15 of 29 patients $(51.7 \%)$ achieved seizure-free outcomes after a mean 30-month follow-up; this rate is lower than what we found in the present study.

Full $\mathrm{HH}$ resection was difficult to achieve. Previous studies showed that $37.9 \%-65.4 \%$ of HHs cannot be completely or almost completely resected. ${ }^{15,17,19}$ The present study found that full resection could not be achieved in $38.5 \%$ of patients, and most of them underwent reoperation. Patients with giant HHs were more likely than those with smaller tumors to receive multiple resections. In our study, as in a study by Pati et al., ${ }^{18}$ reoperation could improve seizure frequency. However, similar to patients who had undergone SRT, most of the RS recipients with unfavorable outcomes had non-GSs as of the last follow-up.

In the present study, we compared the seizure frequency of patients treated with these 2 different surgical methods. Although more RS recipients than SRT recipients achieved favorable outcomes (76.9\% vs $69.2 \%$ ), the difference was not significant. Both SRT and RS recipients showed stable seizure-free rates. Moreover, no significant difference was found between SRT and RS in the rate of repeat procedures $(53.8 \%$ vs $30.8 \%$ with multiple surgeries) after subgroup analysis. SRT is a minimally invasive procedure with better flexibility than RS. Patient 8 had a history of $\mathrm{HH}$ resection but did not achieve seizure reduction, and the patient continued to have both GSs and SPSs. He then came to our center for a minimally invasive approach. After a single SRT procedure, he reached ILAE class 2. For patients with small HHs who are not suitable candidates for RS or refuse to undergo the operation, SRT can provide similar outcomes with a less invasive procedure.

A potential limitation of this study is that patients treated with RS were older, had a longer symptom history, and had larger HHs at baseline than patients who underwent SRT, although these differences were not statistically significant. This likely reflects patient selection for RS or SRT. Patients who are older (possibly with lower surgical risk) and have larger HHs (possibly with greater space-occupying effects) may be more suitable for RS. In addition, we found that intraventricular $\mathrm{HHs}$ are easiest to identify and resect; in these cases, RS can relieve the occupation of the ventricle. In contrast, some small HHs with unclear boundaries are more suitable for SRT than for RS. Moreover, SRT is less invasive than RS and can readily be performed multiple times, which makes SRT the superior choice for children and patients at high surgical risk. Among patients with giant HHs (6 of 26 patients), most were treated with RS (4 of 6 patients). After hamartoma resection, $50 \%$ of patients ( 2 of 4 patients) achieved favorable outcomes, and $25 \%$ of patients ( 1 of 4 patients) reported adverse effects. Encouragingly, both patients with giant $\mathrm{HHs}$ (patient 10,32 $\mathrm{mm}$ and patient 11, $33 \mathrm{~mm}$ ) achieved favorable outcomes after receiving SRT. Although the sample size of patients with giant HHs was small and no significant difference was found in outcomes or adverse effects between SRT and RS ( $p>0.05$, Fisher's exact test), the application of SRT in giant HH deserves further attention.

\section{Adverse Effects of SRT and RS}

Overall, adverse events were recorded in $23.1 \%$ of SRT recipients and $61.5 \%$ of RS recipients, but the difference was not statistically significant $(\mathrm{p}=0.111$, Fisher's exact test). After the initial procedure, only 1 patient $(7.7 \%$ ) with SRT had adverse events, while 8 patients $(61.5 \%)$ with RS reported a variety of side effects $(p=0.011$, Fisher's exact test). There was no significant difference in the incidence 
of adverse effects between the 2 groups (SRT, $42.9 \%$ and RS, 25\%; p > 0.999, Fisher's exact test) after multiple procedures. Given the limited sample size, no predictor was found to be associated with the incidence of adverse reactions after univariate analysis of SRT and RS.

For patients with SRT, hypothyroidism, diminished short-term memory, and weight gain were the major adverse effects. Previous studies rarely reported adverse effects, and when they did, they typically reported that the adverse events improved quickly. ${ }^{22}$ As for hamartoma resection, the most common adverse effect was diabetes insipidus, followed by hypothyroidism and diminished shortterm memory. The side effects of RS have been widely reported by previous studies and include obesity, short-term memory deficit, diabetes insipidus, and hypothyroidism, among others. ${ }^{14,17}$

Generally, patients who underwent SRT had fewer and less-severe adverse events than patients who underwent RS, especially among patients undergoing an initial procedure. SRT provided favorable efficacy and invasiveness with minimal and transient adverse effects.

\section{Other Treatment Options}

Recently, thermocoagulation with laser interstitial thermal therapy (LITT) has compellingly demonstrated a strong therapeutic effect with minimal damage, and this treatment option is increasingly common. ${ }^{28}$ LITT is performed by implanting a laser catheter into the hamartoma and heating it. LITT is technically similar to SRT, and the types of postoperative adverse effects are similar. Previous studies suggested that $84 \%-86 \%$ of patients treated with LITT could achieve freedom from seizures, and the incidence of permanent complications was approximately $8 \%,{ }^{28-31}$ which was similar to the rate observed in SRT. The advantage of LITT over SRT is that a larger volume can be ablated with a single catheter, which might potentially reduce the frequency of repeat heating. Moreover, realtime imaging feedback could improve safety and accuracy. These advantages support LITT as a better choice than SRT. However, with a limited number of studies on LITT, additional patient outcomes must be obtained through long-term follow-up to further explore the efficacy and safety of this treatment. In addition, as a new technology, LITT remains unavailable in many regions. At medical institutions that are not equipped for LITT, SRT can be accomplished using basic stereotactic placement techniques and a radiofrequency lesion generator.

\section{Limitations}

As most of the included patients had small HHs, our results might be more applicable to patients with small $\mathrm{HHs}$ than to those with larger tumors. Additionally, the retrospective nature of this analysis imposes some inherent limitations. Not all variables can be fully controlled; for example, there is potential disparity in patient selection for the treatments, and there may be inconsistencies in the dates of follow-up visits. Additionally, single-center studies are limited in their ability to identify variables that predict outcomes; multicenter collaboration is necessary for a more thorough exploration.

\section{Conclusions}

For patients with small HHs, SRT can provide similar seizure outcomes to RS with a less invasive procedure. Patients who underwent SRT experienced fewer and lesssevere adverse effects than patients who had RS. Patients with giant $\mathrm{HHs}$ (maximum $\mathrm{HH}$ diameter more than 20 $\mathrm{mm}$ ) were more likely to receive multiple $\mathrm{HH}$ resections than those with smaller tumors.

\section{References}

1. Fenoglio KA, Wu J, Kim DY, et al. Hypothalamic hamartoma: basic mechanisms of intrinsic epileptogenesis. Semin Pediatr Neurol. 2007;14(2):51-59.

2. Kameyama S, Masuda H, Murakami H. Ictogenesis and symptomatogenesis of gelastic seizures in hypothalamic hamartomas: an ictal SPECT study. Epilepsia. 2010;51(11):2270-2279.

3. Kerrigan JF, Parsons A, Tsang C, et al. Hypothalamic hamartoma: neuropathology and epileptogenesis. Epilepsia. 2017;58(suppl 2):22-31.

4. Munari C, Kahane P, Francione S, et al. Role of the hypothalamic hamartoma in the genesis of gelastic fits (a videostereo-EEG study). Electroencephalogr Clin Neurophysiol. 1995;95(3):154-160.

5. Errichiello L, Striano P, Galletta D, Striano S. Psychiatric features in gelastic epilepsy and hypothalamic hamartoma: long-term psychodiagnostic observations. Neurol Sci. 2014;35(3):469-471.

6. Harrison VS, Oatman O, Kerrigan JF. Hypothalamic hamartoma with epilepsy: review of endocrine comorbidity. Epilepsia. 2017;58(suppl 2):50-59.

7. Striano S, Santulli L, Ianniciello M, et al. The gelastic seizures-hypothalamic hamartoma syndrome: facts, hypotheses, and perspectives. Epilepsy Behav. 2012;24(1):7-13.

8. Striano S, Striano P. Clinical features and evolution of the gelastic seizures-hypothalamic hamartoma syndrome. Epilepsia. 2017;58(suppl 2):12-15.

9. Striano S, Striano P, Sarappa C, Boccella P. The clinical spectrum and natural history of gelastic epilepsy-hypothalamic hamartoma syndrome. Seizure. 2005;14(4):232-239.

10. Castro LH, Ferreira LK, Teles LR, et al. Epilepsy syndromes associated with hypothalamic hamartomas. Seizure. 2007;16(1):50-58.

11. Oehl B, Brandt A, Fauser S, et al. Semiologic aspects of epileptic seizures in 31 patients with hypothalamic hamartoma. Epilepsia. 2010;51(10):2116-2123.

12. Cross JH, Spoudeas H. Medical management and antiepileptic drugs in hypothalamic hamartoma. Epilepsia. 2017;58(suppl 2):16-21.

13. Choi JU, Kim DS. Treatment modalities for intractable epilepsy in hypothalamic hamartoma. Adv Tech Stand Neurosurg. 2012;39:117-130.

14. Fohlen M, Lellouch A, Delalande O. Hypothalamic hamartoma with refractory epilepsy: surgical procedures and results in 18 patients. Epileptic Disord. 2003;5(4):267-273.

15. Harvey AS, Freeman JL, Berkovic SF, Rosenfeld JV. Transcallosal resection of hypothalamic hamartomas in patients with intractable epilepsy. Epileptic Disord. 2003;5(4):257-265.

16. Ng YT, Rekate HL. Endoscopic resection of hypothalamic hamartoma for refractory epilepsy: preliminary report. Semin Pediatr Neurol. 2007;14(2):99-105.

17. Ng YT, Rekate HL, Prenger EC, et al. Transcallosal resection of hypothalamic hamartoma for intractable epilepsy. Epilepsia. 2006;47(7):1192-1202.

18. Pati S, Abla AA, Rekate HL, Ng YT. Repeat surgery for hypothalamic hamartoma in refractory epilepsy. Neurosurg Focus. 2011;30(2):E3. 
19. Li CD, Luo SQ, Tang J, et al. Classification of hypothalamic hamartoma and prognostic factors for surgical outcome. Acta Neurol Scand. 2014;130(1):18-26.

20. Kameyama S, Murakami H, Masuda H, Sugiyama I. Minimally invasive magnetic resonance imaging-guided stereotactic radiofrequency thermocoagulation for epileptogenic hypothalamic hamartomas. Neurosurgery. 2009;65(3):438449.

21. Kameyama S, Shirozu H, Masuda H, et al. MRI-guided stereotactic radiofrequency thermocoagulation for 100 hypothalamic hamartomas. J Neurosurg. 2016;124(5):1503-1512.

22. Shirozu H, Masuda H, Ito Y, et al. Stereotactic radiofrequency thermocoagulation for giant hypothalamic hamartoma. $J$ Neurosurg. 2016;125(4):812-821.

23. Sonoda M, Masuda H, Shirozu H, et al. Predictors of cognitive function in patients with hypothalamic hamartoma following stereotactic radiofrequency thermocoagulation surgery. Epilepsia. 2017;58(9):1556-1565.

24. Wieser HG, Blume WT, Fish D, et al. ILAE Commission Report. Proposal for a new classification of outcome with respect to epileptic seizures following epilepsy surgery. Epilepsia. 2001;42(2):282-286.

25. Homma J, Kameyama S, Masuda H, et al. Stereotactic radiofrequency thermocoagulation for hypothalamic hamartoma with intractable gelastic seizures. Epilepsy Res. 2007;76(1):15-21.

26. Wang W, Wang W, Guo X, et al. Hypothalamic hamartoma causing gelastic seizures treated with stereotactic radiofrequency thermocoagulation. Epileptic Disord. 2009;11(4):333338.

27. Tandon V, Chandra PS, Doddamani RS, et al. Stereotactic radiofrequency thermocoagulation of hypothalamic hamartoma using robotic guidance (ROSA) coregistered with O-arm guidance-preliminary technical note. World Neurosurg. 2018;112:267-274.

28. Du VX, Gandhi SV, Rekate HL, Mehta AD. Laser interstitial thermal therapy: a first line treatment for seizures due to hypothalamic hamartoma? Epilepsia. 2017;58(suppl 2):77-84.

29. Brandmeir N, Acharya V, Sather M. Robot assisted stereotactic laser ablation for a radiosurgery resistant hypothalamic hamartoma. Cureus. 2016;8(4):e581.
30. Wilfong AA, Curry DJ. Hypothalamic hamartomas: optimal approach to clinical evaluation and diagnosis. Epilepsia. 2013;54(suppl 9):109-114.

31. Zubkov S, Del Bene VA, MacAllister WS, et al. Disabling amnestic syndrome following stereotactic laser ablation of a hypothalamic hamartoma in a patient with a prior temporal lobectomy. Epilepsy Behav Case Rep. 2015;4:60-62.

\section{Disclosures}

The authors report no conflict of interest concerning the materials or methods used in this study or the findings specified in this paper.

\section{Author Contributions}

Conception and design: Guan, S Wang, Luan. Acquisition of data: Zhao, Li, Zhang, Zhou, M Wang, X Wang, Liu, Ma. Analysis and interpretation of data: S Wang, Li. Drafting the article: S Wang. Critically revising the article: Guan. Reviewed submitted version of manuscript: all authors. Approved the final version of the manuscript on behalf of all authors: Guan. Statistical analysis: S Wang. Administrative/technical/material support: Guan, Zhao, Li, Zhou, Luan. Study supervision: Guan, Luan.

\section{Supplemental Information \\ Online-Only Content}

Supplemental material is available with the online version of the article.

Supplementary Tables S1-S3. https://thejns.org/doi/suppl/ 10.3171/2020.2.JNS193423.

\section{Correspondence}

Yuguang Guan: SanBo Brain Hospital, Capital Medical University, Beijing, China. guanyuguang2013@163.com. 